\title{
The Review on the Dark Sides of Creativity
}

\section{Zhang Guangxi}

College of Economics and Management, Zhejiang University of Technology, Hangzhou, China

\section{Email address:}

gxzhang@zjut.edu.cn

\section{To cite this article:}

Zhang Guangxi. The Review on the Dark Sides of Creativity. Science Innovation. Vol. 4, No. 3, 2016, pp. 135-141. doi: 10.11648/j.si.20160403.13

Received: April 2, 2016; Accepted: May 11, 2016; Published: June 8, 2016

\begin{abstract}
Most studies on creativity concentrate on its positive sides, and explore various ways to improve creativity. However, when we view creativity as an outcome or product, and evaluate creativity in terms of its appropriateness, we can not ignore the moral aspect. From the evil genius in the western culture, and taking advantage of loopholes or playing tricks in the eastern culture, we can find many dark sides of creativity. The intension of research on the dark sides of creativity is not suppressing creativity, but helping us to understand creativity more completely, and preventing harm to individuals or society arising from innovative activities. This paper reviews the concept, psychological mechanisms, and the current research domains of the dark sides of creativity. Finally, the author discuss several future research directions.
\end{abstract}

Keywords: Creativity, Dark Side, Psychological Mechanism, Unethical Behavior

\section{创造力的负效应的研究综述}

\section{张光䂀}

经贸管理学院, 浙江工业大学, 杭州, 中国

\section{邮箱}

gxzhang@zjut. edu.cn

摘要: 大多数关于创造力的研究探索创造力的正面价值, 并从各个角度探讨促进创造力的方法。然而, 当我们将创造 力看作产品并衡量成果的社会价值, 就无法忽视创造力的道德维度。从西方世界的魔鬼天才, 到东方文化中的钻空子 和要小聪明, 现实中关于创造力负效应的例子比比皆是, 值得研究者投入更多的研究资源。对创造力负效应的研究并 不是为了抑制创造力, 而是为了让我们更全面地认识创造力, 并防范创造性的活动对个体或社会造成的伤害。本文回 顾了创造力负效应的概念、心理机制、研究领域, 并探讨了未来研究的发展方向。

关键词：创造力, 负效应, 心理机制, 不道德行为

\section{1. 引言}

在人类文明的进程中, 个体创造力的发挥产生了不朽 的艺术作品、突破性的的科技发明、深刻的理论见解。由 于创造力对社会进步的巨大推动作用, 大多数学者都将创 造力视为一种积极的、值得鼓励的品质。然而, 受到个体 差异和环境变化的影响, 创造力的结果既可能是积极的,
也可能是消极的 [1]。首先, 有创造才能的个体可能将创 造力应用在不恰当的地方, 如钻空子、说谎、犯罪 [2] [3] [4]。艺术家可能为了艺术而艺术, 而不考虑艺术 作品的道德影响; 科学家可能为了取得突破性的创新而忽 视研究活动是否符合普适的社会价值。其次, 创造力的实 施不是没有代价的。实施创造性想法带来的变革和不确定 性可能产生人际关系的冲突 [5] [6]。如果组织文化过于强 
调对规范的服从、对不确定性的规避、对权威的尊重, 高 创造力的个体容易感到不被他人理解和接纳, 而承受了比 普通人更高的心理压力。再次, 即使一些发明最初的动机 是好的, 但也可能产生破坏性的后果 [7]。核武器、生物 病毒、计算机病毒等最初发明的目的都是为了造福社会, 但却在后来的应用中产生了破坏性的影响。在最近一篇关 于创造力的理论回顾中, Anderson等人提出学者们应当将 创造力的正效应和负效应纳入统一的模型, 并整合创造力 的前因、过程、后果 [8]。创造力的负效应是非常有趣的 课题, 但当前的研究还处于萌芽状态, 研究主题较为零散, 有很多值得深入探索的问题。通过查阅近二十年的文献, 本文回顾了创造力负效应的概念、机制和研究现状, 探讨 了未来的研究方向。

\section{2. 创造力负效应的研究领域}

创造力 (creativity) 来源于拉丁语creatus, 意思是 “创造和生产” [9]。对创造力的定义多种多样, 目前大 多数学者倾向于将创造力定义为产生新颖和实用的产品 或过程。早期对创造力的研究认为只有少数有特殊能力的 个体才具有创造力的, 更多侧重研究高创造力个体的认知 和人格特征。从 20 世纪中期后, 学者们开始关注创造力产 生的社会环境因素, 认为创造力并不是少数精英才具有的, 个体创造力可能受制于社会环境而没有得到发挥。大多数 研究关注创造力的积极方面, 而关于创造力负效应的研究 相对滞后。Runco认为如果我们将创造力看成是一种工具, 对工具好坏的判断是价值中性的。但由于创造力在大多数 研究中被构念为一种结果, 我们无法将创造力与社会价值 相隔离, 因此创造力作为结果时的确存在正效应和负效应。 对创造力的研究已经从 “大C创造力” 的范式 (创造力是 小部分个体的重大突破) 逐渐转向了 “小C创造力” 的范 式（创造力是普通个体对日常生活的不断改进） [10], 我 们需要更多关注如何在日常生活中防范创造力的负效应。

\section{1. 创造力与不道德行为}

关于创造力负效应的研究主要集中在创造力和不道 德行为之间的关系, 得出的研究结论还缺乏一致性。不道 德行为是指违反了大众接受的道德准则或行为规范的行 为。创造力更多与不服从、反叛、非传统取向联系在一起, 而道德更多与传统、教条、传统取向相联系, 这意味着至 少在遵循传统方面创造力和道德是冲突的 [11]。Mumford 等人通过对自然科学博士生的调研发现个体的某些创造 性思维技巧与科学决策的道德性之间存在正向联系 [12], 而另一些学者发现创造力会导致不道德的行为的产生 [3] [4] [13]。Claxton, Craft和Gardner指出有些创造力 体现了自我本位、自我放纵、物质主义, 浪费了精神资源 和物质资源 [14]。Baucus, Norton, Baucus和Human提出组 织经常用四种手段来促进个体创造力: 1）鼓励个体打破 规则和标准的程序，2）挑战权威以及避免传统，3）形成 冲突、竞争和压力，4）鼓励冒险。然而上述每一类措施 都会产生一系列道德问题: 哪些规则是可以被打破的? 在 什么情况下可以打破规则? 员工如何以道德的方式挑战
权威？管理者如何处理不符合道德的挑战？管理者如何 采取道德的方式形成冲突、竞争或压力? 在鼓励员工冒险 的同时如何保证道德底线 [15]?

创造力的道德维度。大部分对创造力的定义从创造力 产生的过程、人格、环境、产品这四个方面来解释创造力, 但没有区分创造力产生的积极和消极影响 [16]。虽然创造 力是个体的活动和成果, 但无法脱离社会支持和道德评价。 很多科学技术 (器官移植、克隆) 都体现了创新与道德之 间的两难选择。Runco和Nemiro认为评估创造力时, 不仅 需要评估新颖性和实用性, 还要看创造力背后的意图是否 道德 [17]。无论在艺术、科学、教育学、管理学领域, 人 们都应该关注创造力的道德问题。由于快速的技术和社会 变革加剧了人类面临的多种困境, 我们更有必要关注创造 力的道德维度 [18]。Martin更加明确地提出道德创造力 (moral creativity), 认为主观追求具有良好道德价值 的创造性才是值得鼓励的 [19]。为了区分创造力结果的方 向, 学者们提出了积极创造力 (positive creativity) 和 消极创造力 (negative creativity), 还有学者提出恶意 创造力 (malevolent creativity) 和善意创造力 (benevolent creativity)。Kampylis和Valtanen提出了 一个分析框架, 将创造力的结果分为三个维度 [20]：1） 创造者的意图, 2) 对创造者的影响, 3) 对他人的影响。 这三个维度可以组合为多种情况, 可以囊括之前学者的多 种见解: 如恶意创造力属于创造者的意图是消极的, 对创 造者的影响是有利的, 对他人的影响是有害的。关于创造 力道德维度的研究还处于理论探讨的阶段。如何比较客观 的评价创造力的道德维度? 加入道德维度会不会将原本 清晰的问题复杂化? 这些都是理论家面临的研究难点。

创造力与不诚实。哈佛大学的Gino教授及其团队在 2012和2014发表了两项关于创造力和不诚实之间关系的 研究, 在创造力研究领域引起了不小的轰动。他们认为由 于创造性思维具有发散性和灵活性, 创造力可以帮助个体 遇到难题时创造性地发现问题的漏洞, 创造力还可以帮助 个体对不道德行为想出合理化理由。Gino和Ariely对广告 公司员工进行了调查, 发现处于对创造力需求相对较高的 职位的个体更倾向于作出不道德的行为。在实验中, 研究 者控制了个体的智力水平后发现创造力高的个体在随后 的任务 (知觉测试、计算矩阵、多项选择) 中都表现了更 多不诚实的行为, 夸大了实际取得的成绩。当研究者发现 暂时启动的创造力也增加了被试对任务完成结果的不诚 实报告。如果给予个体对不诚实行为辩解的机会, 无论是 临时启动的创造力还是创造性人格都对说谎有更显著的 增强作用。Gino和Wiltermuth进一步探索了相反的因果关 系, 即不诚实也会增加创造力。实验发现处于诱发说谎条 件下被试比处于控制条件下的被试在有更多的不诚实行 为, 并在随后的远距离联想测验中取得更好的成绩。即便 实验组和控制组完成相同的任务, 只是给予实验组的被试 主观说谎的语言刺激, 实验组的被试也有更多的说谎行为, 并在随后的创造力测验中取得更好的成绩。说谎者的感到 受到的规则束缚减少了, 提高了在创造力测试中的成绩。 值得注意的是Gino等人的研究都是在实验条件下开展的, 虽然研究结果既有趣又令人震惊, 但在真实的生活工作场 景中的外部效度还是有待检验的。 
创造力与钻空子。创造力的应用主要是为了解决问题。 当技术手段日益革新、竞争压力日益加大, 个体也会采取 创造性的手段钻空子。钻空子虽然不道德, 但却不违法。 Wang的研究了创造力和钻空子之间的关系 [13]。文中举了 一个例子: 为了能够进驻一个限制超市规模的社区, 沃尔 玛采取了一个创造性的解决方式: 建立两个相连的独立超 市, 每个的规模都在限制范围内, 这个方案可以巧妙绕过 社区对规模的限制。Wang对90项关于钻空子的新闻进行了 编码分析, 发现人们认为钻空子体现了创造力但本身是不 道德的。通过实验对被试的创造力进行启动后, 被试更容 易找到钻空子的方法。无论研究者是否在实验中强调了道 德的重要性, 启动效应中的创造力依然促进了参与者对钻 空子的选择和对钻空子的积极评价。

\section{2. 创造力与犯罪}

创造力与犯罪的关系可以表现为两种形式：1）创造 者的行为或产品被社会认为是有罪的，2）创造的生活方 式被社会认为是有罪的。比如有些艺术家或者作家被关押 不是由于他们的作品, 而是由于他们的生活方式。英国作 家奥斯卡・王尔德因为同性恋，欧 - 亨利因为负债累累而 被入狱 (Brower \& Stahl, 2011)。犯罪可能是发挥创造力 的副产品, 因为创造力需要突破传统, 而对传统的突破有 可能触犯法律。如艺术家为了艺术有可能违反禁忌, 而社 会大众需要时间来接纳和包容新鲜事物, 因此很多艺术家 都有被监禁的经历 (Brower, 1999; Cropley et al., 2008）。研究表明, 高创造力的个体更容易反社会、忽视 权威、我行我素, 这与罪犯具有相似的特征 (Brower, 1999）。还有一些研究发现高创造力者报告他更容易冲动, 而冲动是犯罪的重要特征 (Brower \& Stahl, 2011)。 Eisenman（1999，2007) 发现尽管很有创造力的犯罪份子 的确存在, 但犯罪份子的创造力并不比常人更高。笔者认 为我们不能武断地认为高创造力的个体一定比一般个体 的犯罪概率更高, 现有的研究证据也不能支持这样的推断, 但在创造力和犯罪之间似乎有某些心理和行为方面的相 似特征, 这恰恰是学者们努力探索但还没有得出清晰结论 的地方。

\section{3. 创造力与心理失调}

有研究表明在躁狂抑郁症和创造力之间存在某些联 系(Kéri，2009; Murray \& Johnson, 2010)。高创造力的 个体一般更爱好交际、保持广泛的社会联系、具有显著的 外向性人格, 而躁狂抑郁症的患者一般来说也比常人更加 外向。创造力通常与积极情绪联系在一起, 而高度兴奋是 躁狂抑郁症患者的主要特征。不仅兴奋可能导致患者表现 出更高的创造力, 抑郁也可以帮助患者产生深刻的见解和 体验, 因此在躁狂抑有症的发作阶段患者可以产生更丰富 的艺术创作 (Murray \& Johnson, 2010; Srivastava et al., 2010)。大多数关于精神病与创造力之间关系的研究都是 基于临床研究的选择性小样本, 因此研究者还不能确定这 些结论是否能适用于普通个体。Tremblay, den Nieuwenboer和Kish-Gephart (2010) 对 20,861名成人的调 查表明在有躁狂抑郁症特征的个体在职业选择方面更倾
向选择需求高度创造力的职业 (如美术家、音乐家、作家), 这类职业可以帮助他们保持相对自由并避免遵守严格的 控制。Vellante 等人 (2011) 选取普通大学生样本发现在 创造性成就量表 (CAQ) 上得分高的个体在情感气质自评量 表 (TEMPS-A) 中的某些子量表 (易怒、循环性精神病、情 感增盛）中的得分更高。

\section{4. 创造力与人际冲突}

高创造力的个体内心反叛规则, 他们努力让周围的人 接受他们的想法（Brower \& Stahl，2011）。然而社会中 大部分人总是服从传统、习惯和固定思维, 创新者的思维 偏离和行为偏离有可能在团队中造成人际冲突和任务冲 突。早期的心理学家发现创造力强的个体展现出更多的破 坏性行为 (Scott, 1999), 更加自大 (Chan \& Chan, 1999), 有明显的神经质特征 (Davis，2011), 这些负面的品质可 能加剧高创造力个体人际关系的紧张 (Li, Poon, Tong, \& Lau，2013)。根据同质性原则 (McPherson, Smith-Lovin, \& Cook，2001), 有相似特征的个体容易沟通和合作, 容 易形成稳定的社会交换关系。在一个社会中, 适应者总是 占大多数的, 因此创新者和适应者之间的差异性会造成信 息歪曲、沟通困难, 这将妨碍亲密关系的建立 (Janssen et al., 2004)。当创新者和适应者一起工作时, 创新者偏离 现有标准, 而适应者倡导稳定的秩序 (Kirton, 1976), 两 类人之间的差异是产生冲突的根源。Janssen (2003) 发现 创新性的个体更容易在工作中与同事产生冲突。如果工作 本身对创新者的自我认同很重要, 他们与适应者产生冲突 的可能性就更大, 与同事的冲突降低了创新者的工作满意 感。然而, 笔者认为对于Janssen (2003) 的发现需要辩证 地看待, 因为同事并不总是抵触变革的, 他们也可能欢迎 创造性的想法, 特别是组织面临困难或处于新形势中。

\section{3. 创造力负效应的心理机制}

创造力的负效应主要体现在将创造力用在不道德的 领域, 或者创造力实现的过程对个体的心理健康产生了消 极影响。高创造力的个体不遵循传统、有自己的想法、敢 于冒险、不接受外部的约束 [21]。教育心理学家发现叛逆 与创造力的正向联系在儿童样本中较为明显, 那些在家里 排行中间的孩子一般来说更为叛逆并更有创造力 [22]。

从思维特点上来看, 高创造力的个体具有较强的发散 性思维 [23], 解决问题时采取思维变异 [24]。发散性思维 帮助个体在未产生联系的认知元素之间进行重新组合, 思 维的变异帮助个体打破常规并取得意想不到的发现 [25]。 发散性思维和思维变异一方面可以帮助个体跳僵化的思 维框架, 但也意味着风险、失败、不确定, 以及不符合传 统道德观念的结果出现 [26]。因此, 发散性思维和思维变 异需要适当放松对道德标准的坚持, 甚至超出道德约束。 而遵守道德要求个体服从规则、采取聚合性思维, 这显然 与创造性思维方式存在潜在冲突。创造性个体还具有思维 灵活的特征, 一旦创造性的思维过程启动后, 个体就更容 易发现不道德的解决方案, 并且出于自我保护不道德的行 为进行合理化解释。 
从人格特征上来看, 高创造力者的主观心理状态未 必如我们所期望的那样健康。学者们曾经猜测高创造力 个体的认知过程与精神病人群有相似之处 [27]。从认知 特点上来看, 高创造力的个体善于捕捉看似不相关事物 之间的联系，善于跳出现有框架看问题，这种信息加工 模式需要降低大脑的潜在抑制水平。潜在抑制是指大脑 对当前的刺激不是立即加工而是要等待一些时间, 而降 低的潜在抑制可以提高个体的感受性、人格的开放性、 思维的发散性。因此, 学者们认为降低的潜在抑制水平 可以增加创造力, 而降低的潜在抑制水平也伴随着神经 病理学特征的出现 [28]]。在临床心理研究中, 研究者发 现极有创造力的个体更容易存在情绪失调 [29]、精神状 态不正常 [30]、躁狂抑郁症 [31]。Kéri 发现在neuregulin 1 基因中表现为 $\mathrm{T} / \mathrm{T}$ 基因型的个体智力更高并取得更加 出色学术成就, 而 $\mathrm{T} / \mathrm{T}$ 基因型也曾被发现是与精神病密 切相关的。除了认知过程和基因方面的机制, 高创造力 者的个性和行为特征也可能为自身带来消极影响 [32]。 Kirton将高创造力的个体称为“创新者”(innovators), 而将低创造力的个体称为 “适应者” (adaptors) [33]。 创新者努力摆脱过去和既有理论的制约, 对人际关系不 敏感, 可能威胁团队的和谐。适应者试图解决问题而非 发现问题, 保持稳定和合作对他们很重要。Janssen认为 创新者和适应者之间产生冲突是不可避免的, 因此创新 者很可能要承受不和谐的人际关系、组织的不接纳、较 高的心理压力 [34]。

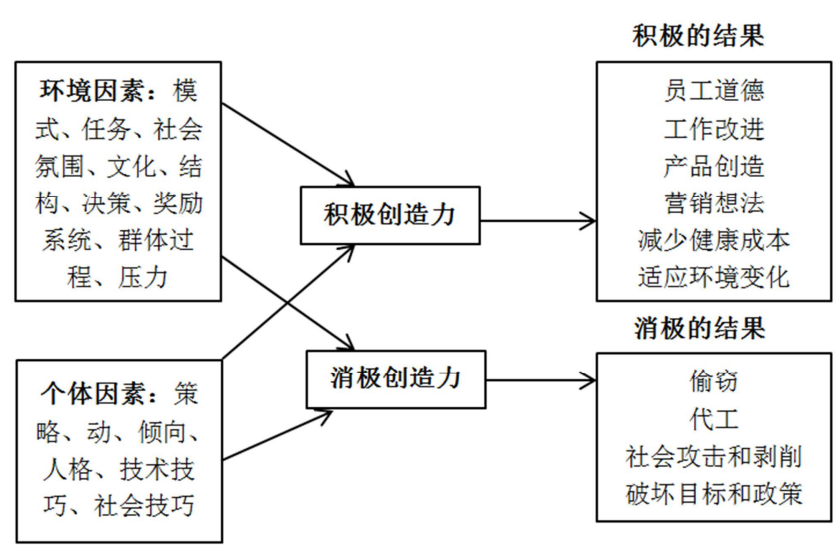

图1 James等人 (1999) 的模型。

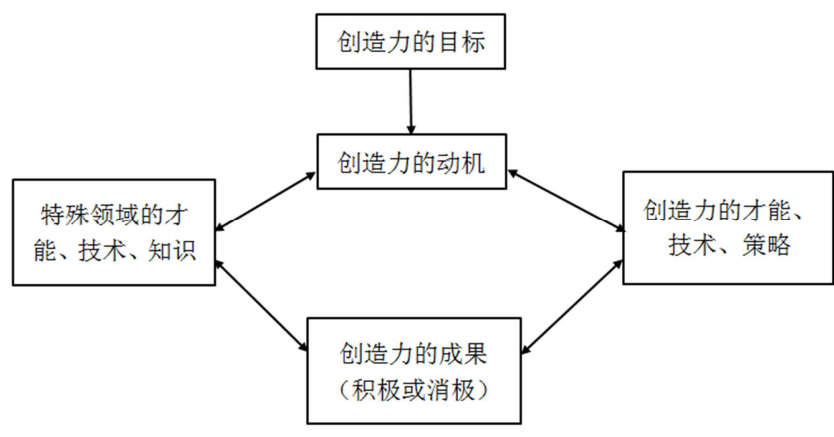

图2 James和Taylor (2010) 的模型。

笔者认为创造力的过程需要大量的认知努力, 但过程 和结果都充满了不确定, 可能产生的成功或失败 [35]。因
此如果大多数研究将创造力定义为过程和结果, 创造力的 负效应的确是存在的。即便学者将创造力定义为人格特征, 创造性人格也可能伴随着消极的后果（如不诚实、精神疾 病）。在认识到创造力转化方向的不确定性后, 学者们试 图将创造力的正负效应整合在统一的模型中。图1是James, Clark和 Cropanzano提出的模型 [1]。这个模型主要用于 分析在组织中影响个体创造力向积极或消极方面转化的 因素, 以及积极创造力和消极创造力在组织中的表现形式。 他们认为积极创造力和消极创造力的内在动力是不一样 的, 消极的情绪和消极的目标将促进个体创造力产生消极 的结果。James和Taylor 对Amabile (1996) 的三因素模型进 行了修订（图2） [36], 在模型中补充了创造力的目标。 他们认为创造力的目标影响了创造力的动机, 然后才影响 创造力的结果。根据目标设定理论, 目标对创造力的影响 有四种机制: 第一, 将注意力和精力分配到创造性活动中; 第二, 能量激活的作用（高目标使个体更努力）; 第三, 使个体对创造活动持之以恒; 第四, 通过利用与任务相关 的知识或策略对创造力产生间接作用 [37]。

\section{4. 未来的研究方向}

\section{1 . 认识创造力的道德维度}

创造力的最常用的维度是新颖性和实用性 [38]。道德 是否应为创造力的第三个维度? 道德困境在很多科学领 域中都普遍存在, 如器官移植、DNA研究、原子能技术。 克隆人技术是一项非常有创造力的技术, 但却被禁止实施, 因为克隆人技术违背人类的伦理道德。因此, Runco和 Nemiro提出道德应成为衡量创造力的第三个维度 [17]。目 前关于道德和创造力的研究还以理论探索、个案研究、以 及实验室研究为主, 未来研究者可以探讨以下的研究问题: 不同文化背景下人们对创造力结果的道德评价有何差异? 在怎样的条件下高创造力的个体更容易作出不道德的行 为? 教育者如何在创造力训练中增加个体对道德问题的 关注?

\section{2. 创造力与社会疏远}

根据同质性原则 [39], 高创造力的个体很可能更容易 体验到社会疏远。社会疏远 (social alienation) 指个体 感到在身体和精神方面与社会脱离的心理状态。社会疏远 是一种消极的心理状态, 会降低个体的情感承诺、工作满 意感、职业动机、工作努力程度]。社会疏远感最初是由 马克思提出的。由于工业革命将人变成生产机器, 蓝领工 人感到社会疏远是普遍的。随后的研究者发现不仅蓝领工 人容易感受到社会疏远, 管理者和知识型工作者同样面临 社会疏远的困扰 [40] [41]。笔者认为高创造力者很可能比 普通个体更容易感到社会疏远。首先, 创新者毕竟是组织 中的少数, 大多数适应者总是倾向于抵制变革。其次, 组 织变革相对个体的改变是很缓慢的。因此, 创造性的想法 很多时候无法得到实施。创新者可能发觉他们的创造力没 有发挥的空间, 工作缺乏意义 [42], 由此产生了社会疏远 感。 


\section{3. 创造力负向转化的边界条件和中介机制}

关于创造力和不道德行为之间关系的机制主要建立 在创造力所需的发散性思维和思维的灵活性的理论基础 上。然而思维的发散既可以产生不道德的行为也可以产生 道德的行为, 在什么条件下创造力会导致不道德行为的产 生? Gino和Ariely从个体因素寻找原因, 认为那些善于为 不道德行为辩解的人更容易将创造力用于不道德领域。然 而仅仅关注个体因素是不够的, 因为创造力的产生和应用 都是社会化的过程, 应当结合社会条件因素进行分析。这 些社会条件因素可以是领导的原因 (侮虐型的领导、不道 德的领导、变革性领导), 可以是社会网络的原因（员工 自身的网络位置影响了道德认同和受到的监督）, 可以是 社会制度的原因 (制度通过制定游戏的规则影响了个体的 道德决策）。在中介机制方面, 自我损耗可能是导致创造 力负向转化的中介变量。自我损耗（ego depletion）指 个体缺少通常所有的资源的一种状态。研究发现如果个体 处于损耗状态, 那么自我控制会受到损耗, 个体从事不道 德行为的概率增加 [43]。处于自我损耗状态的个体容易出 现不良的人际关系, 难以应对关系冲突, 对抵制不道德行 为诱惑的控制能力下降。我们可以推论从事创造性活动的 个体需要搜索大量的信息并对信息进行加工, 消耗了大量 的智力资源, 因此创造力高的个体更容易出现自我损耗的 状态，而自我损耗容易导致不道德行为的出现。对创造力 负向转化的边界条件以及中介机制的研究可能成为新的 研究热点, 可以完善关于创造力负效应的理论。

\section{4. 组织情境下创造力的负效应}

关于创造力负效应的研究以采用实验研究和个案研 究为主, 在组织中的访谈和问卷调查较少。实验室研究虽 然严谨、可以判别因果关系, 但实验条件下的被试表现未 必能代表真实情形下个体的反应。因此, 对组织中员工和 管理者创造力负效应的研究有助于我们更好地揭示影响 负效应的社会情境。既然高创造力的个体思维更灵活, 更 容易偏离规范, 那么怎样的组织环境将促使个体创造力被 应用在不道德的方面? 组织的道德氛围、行为规范、直接 上级的领导方式都可能影响员工的创造力目标, 然后影响 到创造力的动机和创造力的结果。如果一个企业的文化是 急功近利、忽视社会责任, 那么员工很可能将创造力用于 如何欺骗客户而谋求私利, 管理者想方设法将生产成本转 嫁给社会。如果直接上级的领导方式是缺乏道德的, 那么 由此产生的负面情绪和不满很可能产生对组织的报复行 为。如果个体的创造力在组织中不能受到认可, 个体会减 少工作参与度、表现出社会偷闲。到目前为止, 我们对组 织中的个体创造如何产生负效应, 怎样的情形下产生负效 应还不清楚。

\section{5. 中国背景下的本土研究}

不同文化背景影响了创造力受到支持的程度。支持创 造力的文化应该是鼓励自由、容忍不确定性、权力距离较 低 [44]，而中国文化恰恰体现了服从权威、集体主义、规 避风险的传统。无论是个人还是集体在处理问题中求同多
于求异, 因此高创造力的个体在中国文化背景下可能要承 受比在西方文化背景下更多的社会一致性压力。中国文化 中一些要素被认为是抑制创造力的 (如集体主义和权力距 离), 也有一些要素被认为是有利于创造力的 (如“关系”)。 学者们对一些具有中国特色的文化 (如 “中庸”) 对创造 力的影响存在不同的看法。有学者认为创造力的产生需要 突破习惯思维的禁锢, 因而与中庸存在一定的矛盾 [45]。 另一些学者认为人们对中庸存在误解, 中庸体现个体对理 想之道的追求, 是中国传统文化中创造力的体现 [46]。因 此, 关于中国文化对创造力的影响还存在很多模糊不清的 地方, 关于创造力的负效应研究也特别适合在中国的文化 背景下开展。

\section{致谢}

本文得到以下基金的资助: 浙江省哲学社会科学规划 课题 (14NDJC162YB), 国家自然科学基金 (71502167), 浙 江省软科学项目（2015C35010），浙江省自然科学基金 (LQ16G020010)，浙江省 “钱江人才计划” C 类项目 (QJC1402011)，教育部人文社会科学项目（14YJCZH201）, 浙江省教育厅项目（Y201430901）, 浙江省之江青年课题 成果。

\section{参考文献}

[1] James, K., Clark, K., \& Cropanzano, R. (1999). Positive and negative creativity in groups, institutions, and organizations: A model and theoretical extension. Creativity Research Journal, 12(3), $211-226$.

[2] Cropley, D. H. , Cropley, A. J., Kaufman, J. C. , \& Runco, M. A. (Eds.). (2010). The dark side of creativity. New York: Cambridge University Press.

[3] Gino, F. \& Ariely, D. (2012). The dark side of creativity: Original thinkers can be more dishonest. Journal of Personality and Social Psychology, 102(3), $445-459$.

[4] Gino, F. \& Wiltermuth, S. S. (2014). Evil genius? How dishonesty can lead to greater creativity. Psychological Science, 25(4), $973-981$.

[5] Janssen, 0. (2003). Innovative behaviour and job involvement at the price of conflict and less satisfactory relations with co-workers. Journal of Occupational and Organizational Psychology, 76, $347-364$.

[6] Janssen, 0., van de Vliert, E. , \& West, M. (2004). The bright and dark sides of individual and group innovation: a Special Issue introduction. Journal of Organizational Behavior, 25(2), $129-145$. 
[7] Moran, S. (2012). The Dark Side of Creativity. Psychology of Aesthetics Creativity and the Arts, $6(3), 295-296$.

[8] Anderson, N., Potočnik, K., \& Zhou, J. (2014). Innovation and creativity in organizations: A state-of-the-science review, prospective commentary, and guiding framework. Journal of Management, 40(5), $1297-1333$.

[9] Runco, P. G. \& Valtanen, J. (2010). Redefining creativity - analyzing definitions, collocations, and consequences. Journal of Creative Behavior, 44(3), $191-214$.

[10] Simonton, D. K. (2012). Teaching creativity: Current findings, trends, and controversies in the psychology of creativity. Teaching of Psychology, 39(3): 217 222 .

[11] Runco, M. A. (1993). Moral creativity: Intentional and unconventional. Creativity Research Journal, 6, $17-28$.

[12] Mumford, M. D., Waples, E. P., Antes, A. L., Brown, R. P., Connelly, S., Murphy, S. T., \& Devenport, L. D. (2010). Creativity and ethics: The relationship of creative and ethical problem-solving. Creativity Research Journal, 22(1), 74-89.

[13] Wang, L. (2011). The dark side of creativity (Doctoral dissertation, Northwestern University). Retrived from

http://search. proquest. com. eproxy2. 1ib. hku. hk/diss ertations/docview/872077288/459B9B1A10014DA8PQ/1?a c countid=14548.

[14] Claxton, G., Craft, A., \& Gardner, H. (2008). Concluding thoughts: good thinking - education for wise creativity. In A. Craft, H. Gardner \& G. Claxton (Eds.), Creativity, wisdom, and trusteeship: exploring the role of education (pp. 168-176). Thousand Oaks, Calif.: Corwin Press.

[15] Baucus, M. S., Norton, W. I., Baucus, D. A. , \& Human, S. E. (2008). Fostering creativity and innovation without encouraging unethical behavior. Journal of Business Ethics, 81(1), $97-115$.

[16] Runco, M. A. (2004). Creativity. Annual Review of Psychology, 55, $657-687$.

[17] Runco, M. A. \& Nemiro, J. (2003). Creativity in the moral domain: Integration and implications. Creativity Research Journal, 15(1), 91-105.

[18] Gruber, H. E. (1993). Creativity in the moral domain: Ought implies can implies create. Creativity Research Journal, 6(1\&2), $3-15$.
[19] Martin, M. W. 2006. Moral creativity in science and engineering. Science and Engineering Ethics, 12(3), $421-433$.

[20] Kampylis, P. G. \& Valtanen, J. 2010. Redefining creativity - analyzing definitions, collocations, and consequences. Journal of Creative Behavior, 44 (3): 191-214.

[21] Sternberg, R. J. \& Lubart, T. I. (1995). Defying the crowd: Cultivating creativity in a culture of conformity. New York, NY: Free Press.

[22] Gaynor, J. L. R. \& Runco, M. A. (1992). Family size, birth-order, age-interval, and the creativity of children. The Journal of Creative Behavior, 26(2), $108-118$.

[23] Guilford, J. P. (1967). The nature of human intelligence. New York: McGraw-Hill

[24] Simonton, D. K. (1999). Origins of Genius: A Psychology of Science. Cambridge: Cambridge University Press.

[25] Campbe11, D. T. (1960). Blind variation and selective retentions in creative thought as in other knowledge processes. Psychological Review, 67(6), $380-400$.

[26] Kneller, G. E. (1965). The Art and Science of Creativity. New York: Holt, Rinehart \& Winston.

[27] Keefe, J. A. \& Magaro, P. A. (1980). Creativity and schizophrenia: An equivalence of cognitive processing. Journal of Abnormal Psychology, 89(3), $390-398$.

[28] Carson, S. H., Peterson, J. B. , \& Higgins, D. M. (2003). Decreased latent inhibition is associated with increased creative achievement in high-functioning individuals. Journal of Personality and Social Psychology, 85(3), $499-506$.

[29] Richards, R. (1990). Everyday creativity, eminent creativity, and health: "Afterview" ; for CRJ Issues on creativity and health. Creativity Research Journal, 3(4), $300-326$.

[30] Eysenck, H. J. (2003). Creativity, personality, and the convergent-divergent continuum. In M. A. Runco (Ed.), Critical Creative Processes (pp. 95-114). Cresski11, NJ: Hampton.

[31] Murray, G. \& Johnson, S. L. 2010. The clinical significance of creativity in bipolar disorder. Clinical Psychology Review, 30(6) : 721-732.

[32] The neuregulin 1 gene is related to creativity in people with high intellectual achievement. Psychological Science, 20(9): 1070-1073. 
[33] Kirton, M. (1976). Adaptors and innovators: A description and measure. Journal of Applied Psychology, 61(5), $622-629$.

[34] Janssen, 0. 2003. Innovative behaviour and job involvement at the price of conflict and less satisfactory relations with co-workers. Journal of Occupational and Organizational Psychology, 76(3): 347-364.

[35] Taylor, A. \& Greve, H. R. (2006). Superman or the fantastic four? Knowledge combination and experience in innovative teams. Academy of Management Journal, 49(4), $723-740$.

[36] James, K. \& Taylor, A. (2010). Positive creativity and negative creativity (and unintended consequence). In D. H. Cropley, A. J. Cropley, J. C. Kaufman \& M. A. Runco (Eds.), The Dark Side of Creativity (pp. 33 56). New York: Cambridge University Press.

[37] Wu, J., Gong, Y., Song, J., \& Zhang, Z. (2014, May). When and how does intrinsic motivation enhance creativity? the mediating role of creativity goals and the moderating role of extrinsic motivation. Paper presented at the 2014 IACMR Conference, Beijing, China.

[38] Amabile, T. M. (1988). A model of creativity and innovation in organizations. Research in Organizational Behavior, 10, $123-167$.
[39] McPherson, M., Smith-Lovin, L., \& Cook, J. M. (2001). Birds of a feather: Homophily in social networks. Annual Review of Sociology, 27, 415-444.

[40] Lang, D. (1985). Preconditions of three types of alienation in young managers and professionals. Journal of Occupational Behavior, 6(3), 171-182.

[41] Nair, N., \& Vohra, N. (2010). An exploration of factors predicting work alienation of knowledge workers. Management Decision, 48(4), 600-615.

[42] Chiaburu, D. S., Diaz, I., \& De Vos, A. (2013). Employee alienation: relationships with careerism and career satisfaction. Journal of Managerial Psychology, 28(1-2), 4-20.

[43] Yam, K. C., Chen, X. P., \& Reynolds, S. J. (2014). Ego depletion and its paradoxical effects on ethical decision making. Organizational Behavior and Human Decision Processes, 124(2), 204-214.

[44] Erez, M. \& Nouri, R. (2010). Creativity: The influence of cultural, social, and work contexts. Management and Organization Review, 6(3), 351-370.

[45] Yao, X., Yang, Q., Dong, N. N., \& Wang, L. (2010). Moderating effect of Zhong Yong on the relationship between creativity and innovation behaviour. Asian Journal of Social Psychology, 13(1), $53-57$.

[46] Niu, W. (2012). Confucian ideology and creativity. The Journal of Creative Behavior, 46(4), 274-284. 